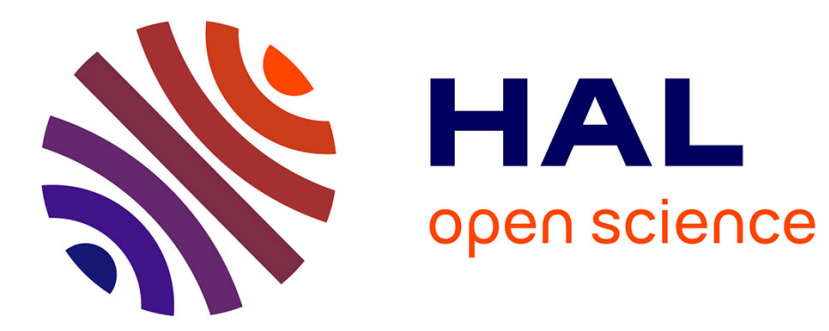

\title{
Nonlinear Dynamics and Chaos in Gas Discharge Systems
}

T. Klinger, F. Greiner, A. Latten, A. Piel, T. Pierre, Gildas Bonhomme, C. Arnas Capeau, G. Bachet, F. Doveil

\section{- To cite this version:}

T. Klinger, F. Greiner, A. Latten, A. Piel, T. Pierre, et al.. Nonlinear Dynamics and Chaos in Gas Discharge Systems. Journal de Physique IV Proceedings, 1995, 05 (C6), pp.C6-131-C6-136. 10.1051/jp4:1995624 . jpa-00253992

\section{HAL Id: jpa-00253992 https://hal.science/jpa-00253992}

Submitted on 1 Jan 1995

HAL is a multi-disciplinary open access archive for the deposit and dissemination of scientific research documents, whether they are published or not. The documents may come from teaching and research institutions in France or abroad, or from public or private research centers.
L'archive ouverte pluridisciplinaire HAL, est destinée au dépôt et à la diffusion de documents scientifiques de niveau recherche, publiés ou non, émanant des établissements d'enseignement et de recherche français ou étrangers, des laboratoires publics ou privés. 


\title{
Nonlinear Dynamics and Chaos in Gas Discharge Systems
}

\author{
T. Klinger, F. Greiner, A. Latten, A. Piel, T. Pierre*, G. Bonhomme*, C. Arnas Capeau**, \\ G. Bachet** and F. Doveil**
}

\author{
Institut für Experimentalphysik Christian-Albrechts-Universität, Olshausenstrasse 40-60, 24098 Kiel, \\ Germany \\ * Laboratoire de Physique des Milieux Ionisés, URA 835 du CNRS, Université Henri Poincaré, \\ B.P. 239, 54506 Vandoeuvre-lès-Nancy cedex, France \\ ** Equipe Turbulence Plasma, Institut Méditerranéen de Technologie, URA 773 du CNRS, Université \\ de Provence, 13451 Marseille cedex 20, France
}

\begin{abstract}
This paper summarizes recent experimental work on nonlinear dynamical phenomena in gas discharge plasmas. As a relatively simple example, chaotic oscillations in low-pressure thermionic discharges are discussed in detail. Period doubling bifurcations and intermittency are clearly identified as standard routes to chaotic behaviour. Most of its dynamical features are well described by simple nonlinear oscillator models. In contrast, plasma waves are spatiotemporal phenomena and thus show a much richer dynamical behaviour. This is demonstrated for currentdriven drift waves in a linear magnetized plasma. Probe arrays provide the required information about the time evolution of the azimuthal wave structure.
\end{abstract}

\section{INTRODUCTION}

The modern concepts of dissipative dynamical systems are now well established in several branches of physics. After pioneering experiments in thermionic discharges [1] and glow discharge tubes [2], these concepts have attracted increasing attention in plasma sciences. In the present paper it is attempted to give an overview of selected experimental work on nonlinear dynamics in plasma waves and oscillations. Special attention is payed to chaos transition scenarios, i.e., examples are given for the most famous routes to chaos: period doubling, intermittency, and quasiperiodicity route [3, 4].

\section{NONLINEAR DYNAMICS OF PLASMA OSCILLATIONS}

Discharge oscillations generally occur as periodically repeated, global reorganizations of an extended plasma region. They are often found in bounded plasma systems ${ }^{1}$ and are inherently nonlinear. The oscillation frequency is in general very low $\left(\omega_{\text {osc }} \ll \omega_{p i}\right)$ and is roughly given by the slow time scale of ion transit in the discharge. Further, plasma oscillations are often characterized by the existence of two different time scales during each period, typical for relaxation oscillators as for example the van der Pol oscillator [5]. The van der Pol oscillator has often served as a useful dynamical model for the understanding of resonance phenomena $[6,7]$ and possesses a surprisingly rich dynamical behavior $[8,9,10]$.

\subsection{Thermionic low-pressure discharges with volume ionization}

The broad class of plasma discharges with thermionic electron emission can be roughly subdivided into systems with surface ionization (Knudsen diode, Q-machine) and systems where ions are produced in the discharge volume (double plasma device, magnetic box). Thermionic discharges with surface ionization have been the subject of extensive experimental, theoretical, and simulation work

\footnotetext{
${ }^{1}$ The boundaries are often electrodes or plasma contactors.
} 
[11]. It has been shown that this discharge geometry is often unstable with respect to the Pierce instability $[12,13]$, a bounded-plasma version of the beam-plasma instability. The Pierce instability is the trigger mechanism for strongly nonlinear low-frequency plasma oscillations, the so-called "potential relaxation oscillation" $[14,15]$. Recently, many of these concepts have turned out to be of great value for the understanding of the dynamics of the more complicated thermionic discharges with volume ionization $[16,17]$.

\subsection{Stable and unstable discharge states}

A schematic diagram of thermionic discharges with volume ionization is shown in Fig. 1(a). Electrons are emitted from the cathode with a half-maxwellian distribution function and are accelerated by the electric field between cathode and anode. If the ionization threshold of the filling gas (argon in the present experiment) is exceeded, ions are produced by electron-neutral collisions. The externally applied discharge voltage $U_{d}$ can be modulated to study the dynamical behaviour of the periodically forced system.

Two stable discharge modes exist, the anode glow mode (AGM) and the temperature limited mode (TLM) $[18,19]$. In Fig. 1(b) the hysteretic discharge voltage/discharge current characteristic is shown together with the plasma potential distribution of the AGM and the TLM. The AGM is characterized

(a)

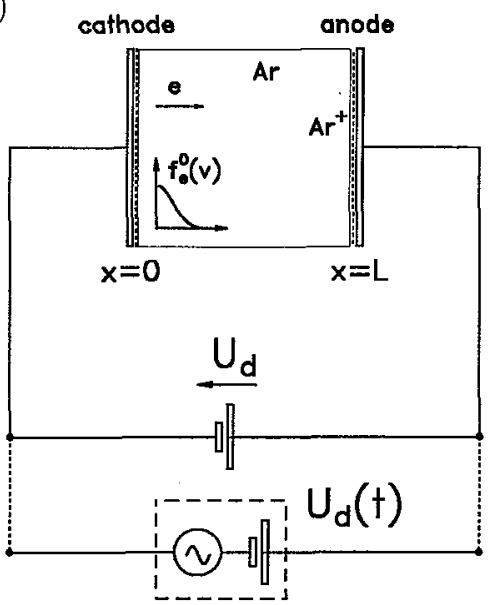

(b)
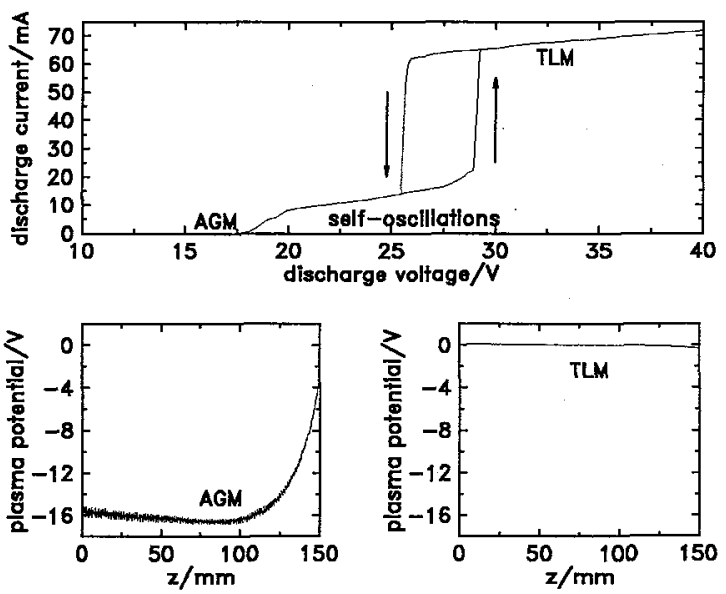

Figure 1: (a) Schematic diagram of thermionic discharges with volume ionization. (b) Hysteresis in the discharge voltage/discharge current characteristic (top), plasma potential structure in the anode glow mode (bottom left) and in the temperature limited mode (bottom right).

(i) by an extended anode sheath where ion production occurs and (ii) by space charge limitation of the electron current. Consequently, the discharge current in the AGM is relatively weak. Above a certain threshold value of the discharge voltage, the AGM becomes unstable and strong relaxation oscillations in the discharge current and in the plasma potential occur (see below). In the TLM, the plasma potential is almost everywhere close to anode potential. The discharge voltage drops in a thin cathode sheath, ionization occurs in the whole discharge volume and the discharge current is only limited by the cathode emission current, i.e, the cathode temperature. The AGM and TLM are discussed in more detail in Ref. [20].

The AGM as well as the TLM becomes unstable in certain regions of the discharge voltage. In both cases, low-frequency relaxation oscillations in almost all plasma parameters are observed, but with different fluctuation levels [Fig. 2]. The oscillation mechanism in the unstable AGM is based on the formation of a transient strong potential double layer that accelerates ions in the negative space 
(a)

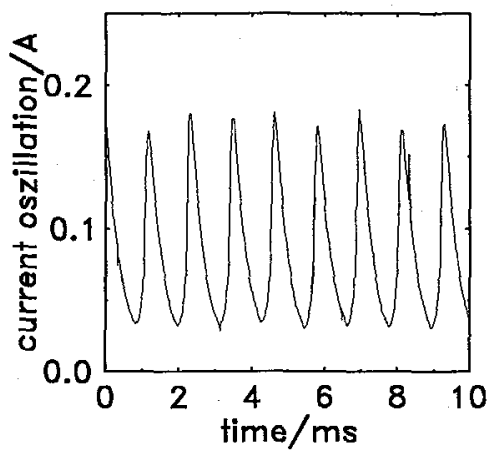

(b)

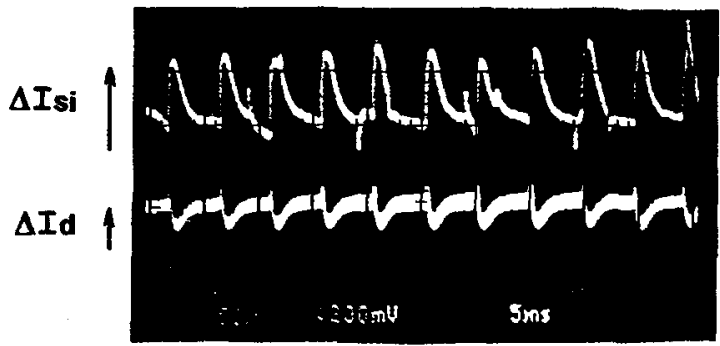

time $\longrightarrow$

Figure 2: (a) Low-frequency relaxation oscillations of the plasma potential in the unstable AGM. The fluctuation level is above $100 \%$. (b) Low-frequency oscillations in the unstable strong-current discharge mode. The fluctuation level is below $5 \%$.

charge region. This process repeats periodically and results in strong discharge current and potential oscillations [21]. The oscillation mechanism is described in detail in Refs. [16, 17]. The oscillation mechanism of the unstable TLM is less well understood. There is evidence for pronounced electron temperature oscillations [22], that can be considered as a hint for an ionization instability [23].

\subsection{Nonlinear dynamics - routes to chaos}

In thermionic discharges, the plasma boundaries play a decisive role for the stability and the dynamics of the plasma [11]. In addition, the boundaries and the external circuit make the (almost collisionless) plasma system become stongly dissipative. For strongly dissipative low-dimensional systems, the period-doubling route and the intermittency route seem to be generic [24]. The experimental findings in thermionic discharges are in agreement with this statement. Fig. 3 shows experimental evidence for
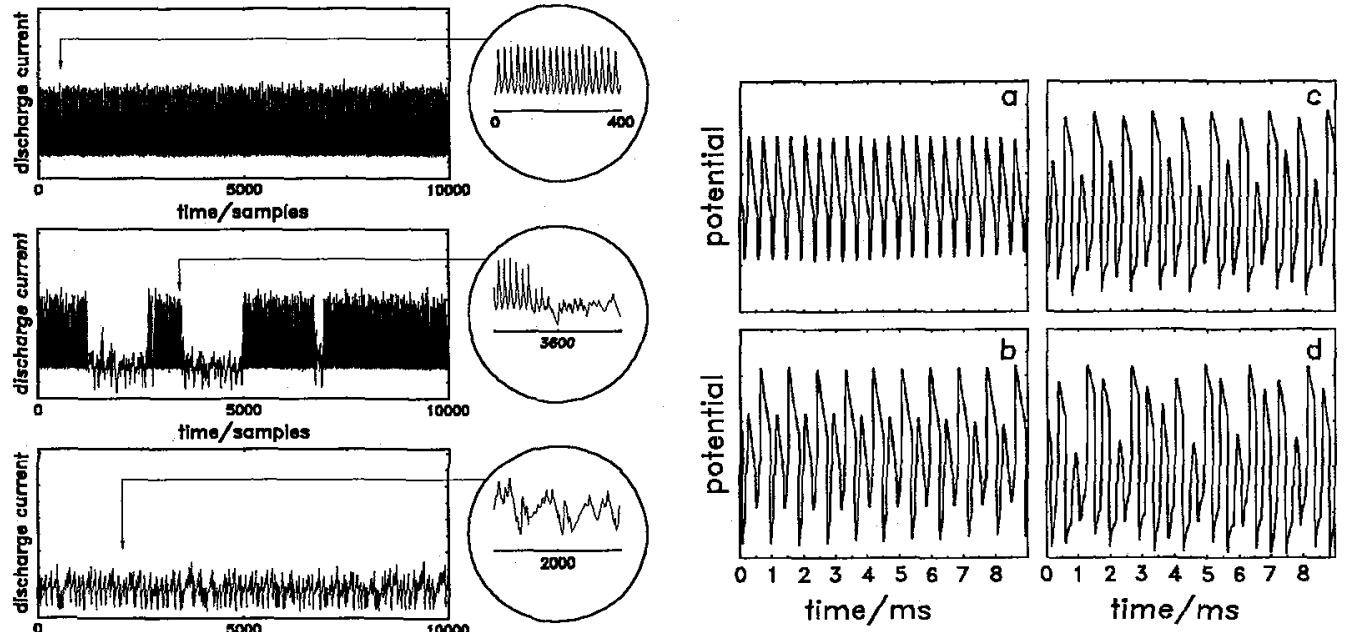

Figure 3: Nonlinear dynamics of the AGM. Left: The undriven system shows the intermittency route to chaos. Right: The periodically driven system performs the period doubling route to chaos, (a) period $1 \rightarrow($ b) period $2 \rightarrow$ (c) period $4 \rightarrow$ period $\infty$. 
the intermittency route (undriven AGM) and the period doubling route (periodically driven AGM) to chaos. Many similar findings have been reported earlier (see for example Refs. [25, 27, 26]) and are in nice agreement with the bifurcation behaviour of periodically driven relaxation oscillators [28]. Furthermore, period doubling bifurcations can be explained physically by the memory effect given by the ions trapped in the potential minimum between anode layer and cathode sheath [21, 17]. It has been shown experimentally that the upper branch oscillations also follow the period doubling route to chaos [22].

\section{NONLINEAR DYNAMICS OF PLASMA WAVES}

In contrast to plasma oscillations, waves in plasmas have the spatial dimension as an additional degree of freedom. The mode structure of the wave may have strong influence on its dynamical features. An example for nonlinear dynamical phenomena and the transition to turbulence in plasma waves, current-driven drift waves [29] in a magnetized triple plasma device [30] are considered. As in case of fluid dynamics experiments [31], the transition to chaos and turbulence suddenly occurs after a finite sequence of Hopf bifurcations [3]. In Fig. 4 power spectra of density fluctuations are shown for different values of the control parameter, the (destabilizing) axial electron drift velocity. After the
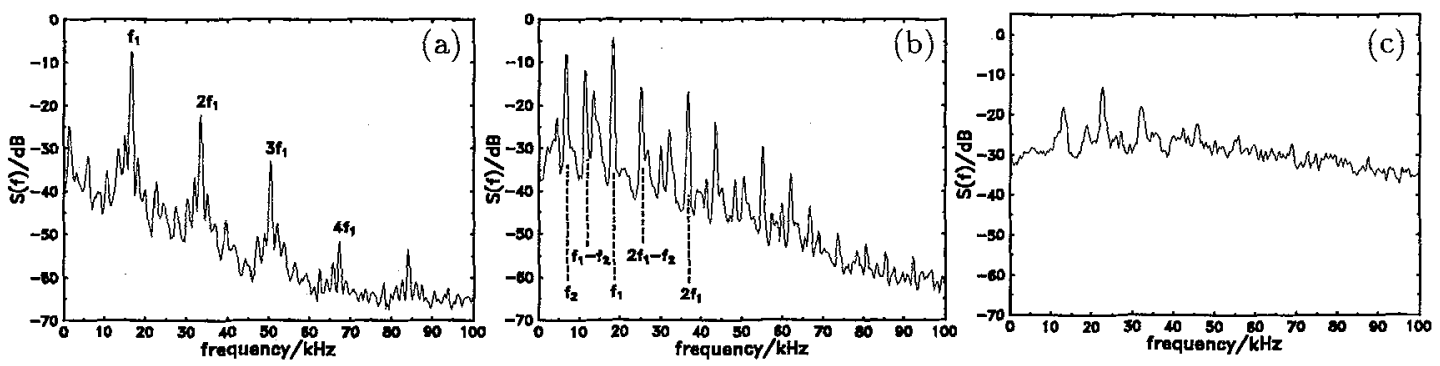

Figure 4: Transition to turbulence via quasiperiodicity. Power spectra of probe signal fluctuations. From left to right: (a) single mode state, (b) two modes with strong nonlinear interaction, (c) fully developed turbulence.

first Hopf bifurcation a single $m=2$ drift mode propagates. The second Hopf bifurcation leads to a state of strongly interacting $m=1$ and $m=2$ modes. (Note the pronounced side bands in the spectrum.) A further increase of the control parameter results in a direct transition to a turbulent state. The fairly complex space-time structure of this state is investigated with an azimuthally arranged Langmuir probe array [32]. This probe array detects instantanously density fluctuations at $N=64$ points of a full azimuthal turn. In this way, the spatiotemporal patterns of regular and turbulent drift waves are obtained. This can be visualized as gray shaded plot or as a sequence of time series [Fig. 5]. The graylevel plot makes evident the complex space-time structure of the turbulent drift waves where large-scale mode structures (appearing as "stripes") interact with smallscale much more localized events. The probe array gives a direct access to the wavenumber spectrum of the drift wave state. The turbulent state shown in Fig. 5 has a broad spectrum with a power-law dependence on the wave number, $S(k) \propto k^{-\alpha}$ [32]. For a detailed understanding of the observed phenomena, however, the spatiotemporal data has to be subject of a variety of advanced numerical anlysis techniques. Besides of conventional Fourier transform and statistical methods, for example biorthogonal decomposition [33] seems to be a promising tool for the analysis of spatiotemporal bifurcations [34].

\section{CONCLUSIONS AND OUTLOOK}

In conclusion, we have briefly described two examples of experimental plasma systems that show many phenomena predicted by the general theory of nonlinear dynamical systems. Beyond the pure 

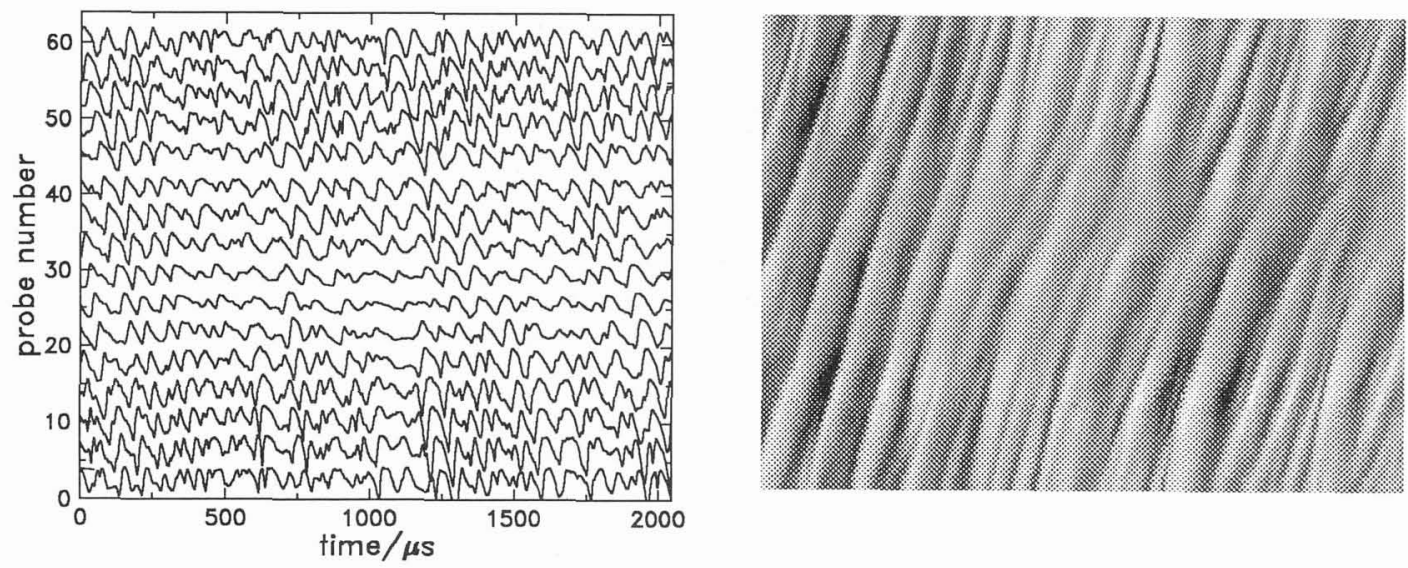

Figure 5: Spatiotemporal structure of turbulent drift waves. Shown are (left) 16 time series of the electron saturation current of equally spaced probes and (right) the same but gray coded plot of the full set of 64 probe signals.

observation, however, it remains the main goal to figure out the physical mechanisms behind. Two future perspectives for a deeper insight into the nonlinear dynamical pheomena in plasmas are (i) a better understanding of the transitions to plasma turbulence (ii) the control of the chaotic state of a plasma, as demonstrated recently for a periodically driven glow discharge [35].

\section{Acknowledgements}

This work was performed under the auspices of the DFG Sonderforschungsbereich 198 project A8 Greifswald-Rostock-Kiel "Kinetik partiell ionisierter Plasmen" and DFG Grant Pi 185/6-1. The french-german cooperation has been supported by the HC\&M contract of the EU (CHRXCT 930356) within the network "Nonlinear phenomena in microphysics of collisionless plasmas: Application to space and laboratory plasmas.".

\section{References}

[1] Cheung P.Y. and Wong A.Y., Phys. Rev. Lett. 59(1987) 551-554.

[2] Braun T. et al, Phys. Rev. Lett. 59 (1987) 613-616.

[3] Jackson E.A., Perspectives of Nonlinear Dynamics, Vol. 1 (Cambridge University Press, Cambridge (UK), 1991).

[4] Jackson E.A., Perspectives of Nonlinear Dynamics, Vol. 2 (Cambridge University Press, Cambridge (UK), 1991).

[5] van der Pol B., Phil. Mag. 3 (1927) 65-80.

[6] Michelsen P. et al., Plasma Phys. 21 (1979) 61-73.

[7] Gyergyek T., Čerček M., Jelić N., and Stanojević M. Phys. Lett. A 177 (1993) 54-60.

[8] Lashinsky H., "Periodic pulling and the transition to turbulence in a system with discrete modes", Symposium on Turbulence of Fluids and Plasmas, Brooklyn, New York, 16-18 April 1968, J. Fox Ed. (Polytechnic Institute of Brooklyn, Polytechnic Press, 1968) pp. 29-46.

[9] Koepke M.E., Alport M.J., Sheridan T.E., Amatucci W.E., and Carroll III, J.J.,, Geophys. Res. Lett. 21 (1994) 1011-1014.

[10] T. Klinger, Greiner F., Rohde A., Piel A., Koepke M.E., Phys. Rev. E 52 (1995) in press.

[11] Kuhn S., Contrib. Plasma Phys. 34 (1994) 495-538. 
[12] Pierce J.R., J. Appl. Phys. 15 (1944) 721-726.

[13] Kuhn S., Phys. Fluids 27 (1984) 1821-1833.

Kuhn S., Phys. Fluids 27 (1984) 1834-1851.

[14] Iizuka S., Michelsen P., Rasmussen J.J., Hatakeyama R., Saeki K., and Sato N., Phys. Rev. Lett. 48 (1982) 145-148.

[15] Bauer F. and Schamel H., Physica D 54 (1992) 235-252.

[16] Klinger T., Greiner F., Rohde A., and Piel A., Phys. Plasmas 2 (1995) 1822-1836.

[17] Greiner F., Klinger T., and Piel A., Phys. Plasmas 2 (1995) 1810-1821.

[18] Malter L., Johnson E.O., and Webster W.M., RCA Rev. 12 (1951) 415-435.

[19] Webster W.M., Johnson E.O., and Malter L., RCA Rev. 13 (1952) 163-182.

[20] Klostermann H., Greiner. F., Klinger T., and Piel A., Plasma Sources Sci. Technol. 3 (1994) 134-141.

[21] Greiner F., Klinger T., Klostermann H., and Piel A., Phys. Rev. Lett. 70 (1993) 3071-3074.

[22] Arnas Capeau C., Bachet G., and Doveil F., J. Plasma Phys. (1995) accepted for publication.

[23] Oleson N.L. and Cooper A.W., Adv. Electron. Electron Phys. 24 (1968) 155-278.

[24] Ott E., Sauer T., and Yorke J.A., Coping with Chaos (Wiley, New York, 1994).

[25] Cheung P.Y, Donovan S., and Wong A.Y., Phys. Rev. Lett. 61 (1988) 1360-1363.

[26] Quin J., Wang L., Yuan D.P., Gao P., Zang B.Z., Phys. Rev. Lett. 63 (1989) 163-166.

[27] Jiang Y., Wang H., Yu C.X., Chinese Phys. Lett. 5 (1988) 201-204.

[28] Mettin R., Parlitz U., and Lauterborn W., Int. J. Bifurcation and Chaos 3 (1993) 1529-1555.

[29] Ellis R.F., Marden-Marshall E., and Majeski R., Plasma Physics 22 (1980) 113-132.

[30] Pierre T., Leclert G., and Braun F., Rev. Sci. Instrum. 58 (1987) 6-11.

[31] Glazier J.A, and Liebchaber A., IEEE Trans. Circ. Sys. 35 (1988) 790-809.

[32] Latten A., Klinger T., Piel A., and Pierre T., Rev. Sci. Instrum. 66 (1995) 3254-3262.

[33] Aubry N., Guyonnet R., and Lima R., J. Nonlinear Sci. 2 (1992) 183-215.

[34] Madon A. and Klinger T., Physica $D(1995)$ accepted for publication.

[35] Weltmann K.D., Klinger T., and Wilke C., Phys. Rev. E 52 (1995) in press. 